\title{
Seeing what they read and hearing what they say: Readers' representation of the story characters' world
}

\author{
Celia M. Kuin ANd April M. Drumm \\ State University of New York, Binghamton, New York
}

\begin{abstract}
Do readers "see" the words that story characters read and "hear" the words that they hear? Just as priming effects are reduced when stimuli are presented cross-modally on two different occasions, we found reduced transfer effects when story characters were described as experiencing stimuli cross-modally. In Experiment 1, a repeated phrase was described as being part of a spoken message in both Story A and Story B, and transfer effects were found. In Experiment 2, in contrast, when the phrase was described as a written note in one story and a spoken message in the other, reading-time results indicated that readers did not retrieve the meaning of the repeated phrase. The results are consistent with findings indicating that visual imagery simulates visual processing and that auditory imagery simulates auditory processing. We conclude that readers mentally simulate the perceptual details involved in story characters' linguistic exchanges.
\end{abstract}

When we are immersed in a novel, to what extent is our experience of the protagonist's world similar to our experience of our own world? Do we see, in our mind's eye, the words that she reads in the morning newspaper? Do we hear, in our mind's ear, the morning greeting that she gives to her daughter? If a protagonist leaves a note for her daughter saying "good morning" rather than speaking the words, is the phrase encoded differently by the reader? More specifically, given that people encode some of the perceptual details of the words that they read and hear, rather than simply extracting their meaning, we ask whether people also encode some of the perceptual details of the words that story characters read and hear.

Research clearly indicates that when a word is repeated in two different modalities on two different occasions, there will be less facilitation in processing the second encounter of the word than there will be if the word was presented in the same modality on both encounters (e.g., Jacoby \& Dallas, 1981; Westerman, Lloyd, \& Miller, 2002). For example, using a variety of implicit-memory tests, Rajaram and Roediger (1993) examined priming effects for visually presented test words. They found reduced priming effects when the initial presentation of the words was auditory, as compared with when it was visual. Although there are a variety of explanations for exactly why this might be the case (see, e.g., Schacter, 1994), these reduced effects indicate that people encode some perceptual details of the words they experience, such as the orthographic or phonological features.

But what happens if a story character is described as experiencing a word in two different modalities? Would a mismatch in the perceptual details of the word, as experienced by the character, reduce the probability that the reader would retrieve the word from memory? To answer this question, we examined repetition effects when a phrase is repeated across two unrelated passages. Previous research on repetition effects (Klin, Drumm, \& Ralano, 2009; Klin, Ralano, \& Weingartner, 2007) has indicated that comprehension of a phrase in Story B is influenced by the meaning of the phrase in Story A. In these experiments, Story A and Story B had different sets of characters, but both described one character, the communicator, leaving the same written note for a second character, the addressee (e.g., "He sure is great company"). In both Story A and Story B, therefore, the addressee experienced the note visually. Under these conditions, the phrase was reactivated from memory when readers encountered it in Story B.

But what if in one of the two passages the phrase is described as having been spoken by the communicator rather than written? For example, instead of the communicator writing a note, he calls the addressee on the telephone. Although it seems likely that readers will infer that the addressee has processed the phonological details of the words in one case and the orthographic details in the other, we ask whether the reader's memory representation of the phrases will include those perceptual details as well. Although the reader's experience is purely visual in both cases, we ask whether the character's experience will make this analogous to a cross-modal task.

Why might we expect the perceptual details of a story character's experience to be stored in the reader's memory? 
There is extensive evidence that readers develop a memory representation of a text that consists of more than the actual words of a text. The representation is much richer and includes aspects of the situation described by the text (see, e.g., Bower \& Morrow, 1990), including details that are consistent with the story characters' experience (see, e.g., O'Brien \& Albrecht, 1992). Given this, readers might include some of the perceptual details of the story character's linguistic experience in their memory representation. Recent research on embodied, or grounded, cognition also provides a framework for this hypothesis. According to this framework, cognition is grounded in perception and bodily action (see, e.g., Barsalou, 1999, 2008; Fischer \& Zwaan, 2008; Gibbs, 2006), and a critical element in understanding other people's behavior involves simulating it. For example, after reading a sentence in which a character performed an action (e.g., "He closed the drawer"), readers were faster to press a key when it involved making a movement in the same direction as the story character's movement (Glenberg \& Kaschak, 2002). Just as readers mentally simulate the perceptual details involved in a character's movements, they might therefore mentally simulate the perceptual details involved in a character's linguistic experience.

\section{EXPERIMENT 1}

We began with a replication and extension of findings from Klin and colleagues (Klin et al., 2009; Klin et al., 2007). In those experiments, participants were presented with experimental items that were made up of two passages-Story A and Story B. (See the Appendix.) Furthermore, there were two versions of Story A: (1) A repeated version, which contained the target, the repetition line; and (2) a not repeated version, which did not. Story B contained the repetition line and was the same for the two conditions. The question of interest involved the processing of the repetition line in Story B: Would readers in the repeated condition experience the phrase as familiar on the basis of having reactivated it from memory?

To answer this question, we examined readers' understanding of the Story B repetition line. Note that this line was ambiguous: In the sample passage, it was unclear whether Justin was being sincere or sarcastic. In the repeated version of Story A, however, the context clearly biased a sarcastic interpretation of this phrase. Assuming there is a transfer effect, when participants read the repeated phrase in Story B they will reactivate the repetition line from Story A as well as their interpretation of it; thus, participants should be more likely to interpret the repetition line in Story B as sarcastic in the repeated version than in the not repeated version. Reading times should, consequently, be shorter on the disambiguation line, which followed the repetition line, because it was consistent with the sarcastic meaning of the repetition line. This is exactly what was found by Klin and colleagues (Klin et al., 2009; Klin et al., 2007).

The present experiment involved a procedure identical to that used by Klin and colleagues (Klin et al., 2009;
Klin et al., 2007). Further, the materials differed by only a few words. Whereas the passages in Klin et al. (2009) and Klin et al. (2007) described the critical repeated phrase as having been part of a note or an e-mail in both Story A and Story B, the critical phrase in the present experiment was described in both Story A and Story B as having been spoken, either in person or on the telephone. This experiment was run for two reasons. First, because Experiment 2 involved a mismatch in modalities - the critical phrase was described as part of a written note in one story and as part of a spoken message in the other-we wanted to ensure that describing the repeated phrase as having been spoken, rather than written, did not somehow reduce its salience or memorability. Second, if the manipulation of modality in Experiment 2 reduced or eliminated the transfer effects, as we hypothesized it would, it is important to have demonstrated that this effect was present when the modalities matched.

Although studies examining the repetition of individual words have often used reading time during the second reading as the dependent measure (e.g., Raney \& Rayner, 1995), the primary dependent measure in the present experiment was reading time on the Story B disambiguation line, rather than the reading time on the repetition line itself. In contrast with encountering a repeated word, which tends to lead to faster reading, encountering a repeated phrase might surprise readers, causing reading times to increase. Consistent with this, Klin and colleagues (Klin et al., 2009; Klin et al., 2007) found variable effects on the repetition line but shorter reading times on the disambiguation line in the repeated condition; thus, reading time on the disambiguation line is a more reliable measure.

\section{Method}

Participants. Thirty-five undergraduates participated. The data from 1 participant who did not follow instructions were eliminated.

Materials. There were 14 experimental items, with each item consisting of a pair of independent stories, Story A and Story B. In the critical information section of the repeated version, the communicator either left a two-sentence sarcastic spoken message for the addressee or had a spoken conversation with the addressee consisting of two sentences of dialogue. The first sentence was the repetition line (e.g., "Damian sure is great company"). This sentence was written so that it would be ambiguous if it appeared in isolation, and thus could be interpreted as either sarcastic or sincere. The context that preceded the repetition line, however, stated explicitly that the message writer was angry or unhappy. Further, the dialogue sentence following the repetition line was clearly sarcastic. In the not repeated version of Story A, the repetition line was not included. In its place, the communicator left a message or had a conversation with the addressee that was unambiguous and sincere. The criticalinformation sections in Story A were matched for length across the two versions.

Story B was identical in the repeated and not repeated conditions. Story B contained the repetition line, which had the same phrase (e.g. " "sure is great company") that the repeated version of Story A did. Further, the phrase was described as having been spoken. In contrast with Story A, however, the repetition line was presented in a neutral context, and it was therefore unclear whether it was intended to be sarcastic or sincere. The repetition line was followed by the disambiguation line, which made it clear that the line was intended sarcastically. 


\section{Results and Discussion}

Statistical analyses were performed both with participants as a random effect variable $\left(t_{1}\right)$ and with items as a random effect variable $\left(t_{2}\right)$. An alpha level of .05 was used for all analyses. The data were trimmed, eliminating $5 \%$ of the data (Tukey, 1977). Reading times on the disambiguation line were $165 \mathrm{msec}$ faster in the repeated condition than in the not repeated condition $\left[t_{1}(33)=2.44, S E M=\right.$ $\left.67.60, d=0.42 ; t_{2}(13)=2.20, S E M=72.93\right]$. This is exactly the pattern of results found in Klin et al. (2009) and Klin et al. (2007) when the repeated phrase was described as having been part of a written note in both Story A and Story B. In contrast, there was no difference in reading time on the repetition line (1,656 vs. $1,660 \mathrm{msec}$ for the repeated and not repeated conditions, respectively). Although faster readings in the repeated version might have been expected, the effect on the repetition line has been quite variable in our previous work. Any savings involved in encountering repeated lexical items may be offset by the process of accessing the sarcastic meaning of the repeated phrase. Consistent with this, there is evidence that figurative meanings are accessed more slowly than are their literal counterparts (see, e.g., Gibbs, 1990). Regardless of the reason for the variability in the repetition-line data, shorter reading times on the disambiguation line in the repeated condition clearly indicate that the words as well as the meaning of the repetition line were retrieved from memory. We can thus conclude that when there is a match, from the perspective of the characters, in the modality of the repetition line-either written/written (Klin et al., 2009; Klin et al., 2007) or spoken/spoken — transfer effects are found.

\section{EXPERIMENTS 2A AND 2B}

In Experiments 2A and 2B, we examined transfer effects when a phrase was described as having been spoken in one story and as written in the other. Note, however, that because the experiment involved reading and not listening, the modality of the linguistic exchange differed only from the point of view of the story characters. We asked whether transfer effects would be reduced because of this change in modality. In Experiment 2A, the critical message was described as written in Story A and as spoken in Story B; in Experiment 2B, the order was reversed. It is important to note that there was virtually no decrease in the amount of lexical overlap in the repetition lines. On average, Story A and Story B in Experiments 2A and 2B shared only one fewer word than did Story A and Story B in Experiment 1. To the extent that we would find any decrease in transfer effects in the present experiment, therefore, it would be attributable to a mismatch in the modality of the two communications, rather than to a mismatch in the lexical items.

\section{Method}

Participants. Sixty-one undergraduates participated in Experiment 2A; the data from 4 participants who did not follow instructions were eliminated. Fifty-four undergraduates participated in Experiment $2 \mathrm{~B}$; the data from 4 participants were eliminated.
Materials. As in Experiment 1, there were 14 experimental items, with each item consisting of a pair of independent stories, Story A and Story B. All aspects of the materials were identical to those from Experiment 1 except for a couple of words describing the critical phrase. In Experiment 2A, Story A was modified to describe the critical phrase as having been part of a written note. In Experiment 2B, Story B was modified in this way. On average, this involved a change in only one word.

Design and Procedure. The design and procedure were identical to those from Experiment 1.

\section{Results and Discussion}

Means were calculated after discarding outliers (Tukey, 1977); this eliminated $6.3 \%$ and $7.9 \%$ of the data in Experiments $2 \mathrm{~A}$ and $2 \mathrm{~B}$, respectively. There was no main effect of experiment; reading times on the disambiguation line were almost identical for Experiments $2 \mathrm{~A}$ and $2 \mathrm{~B}$ $[t(105)=0.30, S E M=89.01, p=.77]$.

In contrast with Experiment 1, there were no differences in reading time between the repeated and not repeated conditions on the disambiguation line. In fact, reading times were almost identical, differing by only $20 \mathrm{msec}$ in Experiment $2 \mathrm{~A}\left[t_{1}(56)=0.38, S E M=54.27, p=.70, d=\right.$ $\left.0.05 ; t_{2}(13)=0.22, S E M=71.02, p=.83\right]$ and $10 \mathrm{msec}$ in Experiment $2 \mathrm{~B}\left[t_{1}(49)=0.17, S E M=60.26, p=.87\right.$, $\left.d=0.02 ; t_{2}(13)=0.46, S E M=81.14, p=.65\right]$. The interaction between these null effects in the mismatched conditions (Experiments 2A and 2B combined) and the 165 -msec effect in the matched condition (Experiment 1) was marginally reliable $[t(139)=1.84, S E M=80.83$, $p=.068, d=0.16]$. (See Figure 1.)

By describing the repetition line as having been spoken in one story and written in the other, a large and robust effect was eliminated. The elimination of the effect is particularly impressive given that the manipulation involved changing only a couple of words. Further, not only were reliable within-modality transfer effects found in Experiment 1 , in which the phrase was described as having been spoken in both Story A and Story B, Klin and colleagues (Klin et al., 2009; Klin et al., 2007) found similarly sized

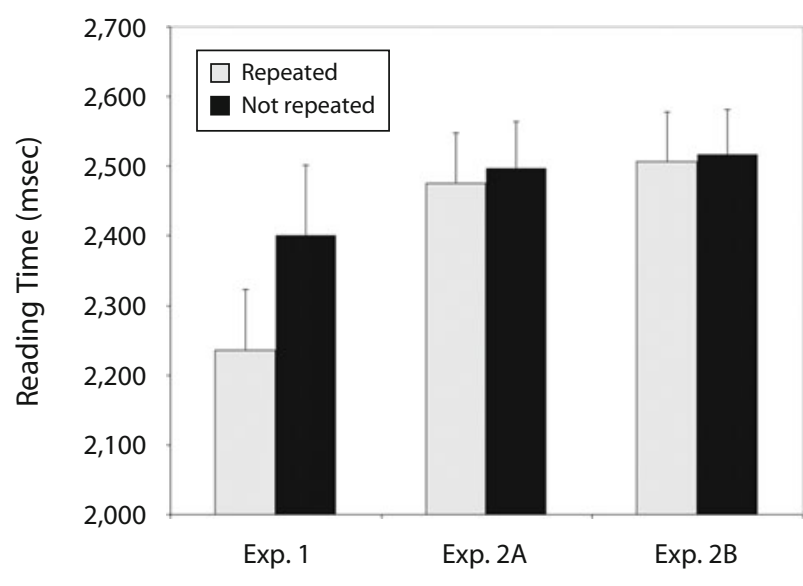

Figure 1. Mean reading times for Story $B$ disambiguation lines for Experiment 1 (spoken/spoken), Experiment 2A (written/ spoken), and Experiment 2B (spoken/written). Error bars represent the standard errors of the means. 
within-modality effects when the phrase was described as having been written in both Story A and Story B. Further, Klin et al. (2009) found transfer effects even when a filler passage intervened between Story A and Story B.

In contrast with the null effect on the disambiguation line in Experiments 2A and 2B, reading times were shorter on the repetition line in the repeated version than on the repetition line in the not repeated version. This difference was reliable in Experiment 2A $\left[t_{1}(56)=3.55, S E M=\right.$ $\left.49.16, d=0.47 ; t_{2}(13)=3.27, S E M=48.94\right]$ and reliable in the subjects analysis in Experiment $2 \mathrm{~B}\left[t_{1}(49)=3.59\right.$, $S E M=48.63, d=0.51 ; t_{2}(13)=1.95, S E M=74.89$, $p=.073]$. These repetition-line effects suggest that the mismatch between Story A and Story B in the modality of the communication did not eliminate the reactivation of the individual lexical items; however, it did eliminate meaning-selection effects - reactivation of the sarcastic, selected meaning of the ambiguous phrase.

\section{GENERAL DISCUSSION}

Decreased cross-modality versus within-modality priming effects indicate that some of the perceptual details of studied words are encoded during study and retrieved at test. Consistent with this finding, neuroimaging evidence indicates that the recall of previously studied pictures involves the activation of visual areas of the brain, whereas the recall of previously studied sounds involves the activation of auditory areas (see, e.g., Wheeler, Petersen, \& Buckner, 2000). Not only have these sensoryspecific effects been found for stimuli that people actually experience, but they also have been reported when people are simply imagining the stimuli: Both behavioral and neural evidence indicates that visual imagery simulates visual processing (see, e.g., Kosslyn, Ganis, \& Thompson, 2001) and that auditory imagery simulates auditory processing (see, e.g., Halpern, Zatorre, Bouffard, \& Johnson, 2004).

Given that a central aspect of reading involves forming images, in the present study we asked about the possible involvement of visual and auditory processing during reading. Most people can recall the layout of a favorite character's home or the cadence of his voice; thus, it seems possible that readers engage in the kinds of visual and auditory simulations that have been demonstrated in imagery studies. We hypothesized that readers include some of the perceptual details of the characters' linguistic experience in their representation, hearing the words that a character hears, or seeing the words that he reads.

After demonstrating within-modality transfer effects for a repeated phrase in Experiment 1, in Experiments 2A and $2 \mathrm{~B}$ these effects were eliminated when the story characters processed the words of the phrase cross-modally. Critically, the elimination of the transfer effects occurred even though the repetition was within a modality for the readers. The participants in the experiments simply read; nothing was ever said or heard. Despite this, there was a strong influence of the modality of the phrase from the point of view of the story character. Furthermore, al- though all of the written conditions involved a character leaving a note, and all of the spoken conditions involved characters speaking, there was no greater lexical overlap in the matched conditions than in the mismatched conditions. There also did not seem to be any greater "scenario" overlap in the matched versus the mismatched conditions; even in the matched conditions, the scenarios varied substantially from Story A to Story B. For example, in the sample passage in Story A, the critical message was left by a dog sitter to a dog owner, whereas in the sample passage in Story B, the critical message was left by a boyfriend for his girlfriend. We are thus able to conclude that the reduced cross-modal effects in the mismatched conditions resulted from readers' encoding some aspects of the perceptual experience of the story characters in their text representation and then using this information during retrieval. When this perceptual information did not match, transfer effects were reduced.

In contrast with the null effect on the disambiguation line in Experiments 2A and 2B, repetition effects were found on the Story B repetition line; participants read the line more quickly in the repeated condition. The change in modality thus did not prevent reactivation of the lexical items of the phrase. Presumably, this was because the lexical items were processed visually by the reader in both Story A and Story B. In this way, the task was very similar to a standard repetition-priming task, making the shorter reading times on the second reading unsurprising. Consistent with this, Klin et al. (2009) found that repetition effects for the lexical items were long lasting and relatively immune to manipulations at the text level. Perhaps we would see a different pattern of results if the perceptual manipulation were not simply imaginary: If participants read Story A but listened to Story B, transfer effects might be reduced on the repetition line itself.

The fact that the modality mismatch influenced reading times only on the disambiguation line suggests that the mismatch had its primary influence on the readers' representation of the situation that was described in the text, rather than on the representation of the individual words of the phrase. Readers represented the phrase either as part of a note or as part of a spoken utterance. Although we cannot definitively specify the format in which this information was represented, it is striking that describing a phrase in a story as spoken instead of as written produced such a significant change in processing. After all, the scenarios surrounding the critical phrase in Stories $\mathrm{A}$ and $\mathrm{B}$ mismatched in a multitude of other ways that did not disrupt retrieval. In the matched conditions in Experiment 1, for example, the critical phrases in Stories A and $\mathrm{B}$ were described as having been spoken by different characters; in Story A the phrase was part of a voicemail, and in Story B it was part of a conversation; in Story A the "great company" referred to a dog, and in Story B it referred to a person. This suggests that the modality of the phrase, from the characters' perspective, was encoded by readers and was salient.

Our findings fit well with recent proposals of embodied cognition in which the text representation is 
perceptual in nature. They could also be explained by traditional accounts of knowledge representations, with knowledge consisting of feature lists or semantic networks. Although the present data cannot resolve fundamental questions about the format in which knowledge is represented, they do tell us that imagining a story character reading and listening is much like imagining ourselves reading and listening, which is much like actually reading and listening. Thus, just as reduced cross-modal priming effects are found when real people read and then listen, they are also found when story characters read and then listen.

\section{AUTHOR NOTE}

Portions of these data were reported at the 18th Annual Meeting of the Society for Text and Discourse. We thank Alicia Kosiba, Steven Kovacs, and John Sentigar for their assistance. We also thank William Levine and Kristin Weingartner for comments on an earlier draft. Correspondence concerning this article should be addressed to C. M. Klin, Department of Psychology, Binghamton University, Binghamton, NY 13902-6000 (e-mail: cklin@binghamton.edu).

\section{REFERENCES}

Barsalou, L. W. (1999). Perceptual symbol systems. Behavioral \& Brain Sciences, 22, 577-660. doi:10.1017/S0140525X99002149

Barsalou, L. W. (2008). Grounded cognition. Annual Review of Psychology, 59, 617-645. doi:10.1146/annurev.psych.59.103006.093639

Bower, G. H., \& Morrow, D. G. (1990). Mental models in narrative comprehension. Science, 247, 44-48. doi:10.1126/science. 2403694

Fischer, M. H., \& ZWAAN, R. A. (2008). Embodied language: A review of the role of the motor system in language comprehension. Quarterly Journal of Experimental Psychology, 61, 825-850.

GibBs, R. W., JR. (1990). Comprehending figurative referential descriptions. Journal of Experimental Psychology: Learning, Memory, \& Cognition, 16, 56-66. doi:10.1037/0278-7393.16.1.56

GiBBS, R. W., JR. (2006). Metaphor interpretation as embodied simulation. Mind \& Language, 21, 434-458. doi:10.1111/j.1468-0017.2006 $.00285 . \mathrm{x}$
Glenberg, A. M., \& KaschaK, M. P. (2002). Grounding language in action. Psychonomic Bulletin \& Review, 9, 558-565.

Halpern, A. R., Zatorre, R. J., Bouffard, M., \& Johnson, J. A. (2004). Behavioral and neural correlates of perceived and imagined musical timbre. Neuropsychologia, 42, 1281-1292. doi:10.1016/ j.neuropsychologia.2003.12.017

JACOBY, L. L., \& Dallas, M. (1981). On the relationship between autobiographical memory and perceptual learning. Journal of Experimental Psychology: General, 110, 306-340. doi:10.1037/0096 $-3445.110 .3 .306$

Klin, C. M., Drumm, A. M., \& Ralano, A. S. (2009). Repeated text in unrelated passages: Repetition versus meaning selection effects Memory \& Cognition, 37, 556-568.

Klin, C. M., Ralano, A. S., \& Weingartner, K. M. (2007). Repeating phrases across unrelated narratives: Evidence of text repetition effects. Memory \& Cognition, 35, 1588-1599.

Kosslyn, S. M., Ganis, G., \& Thompson, W. L. (2001). Neural foundations of imagery. Nature Reviews Neuroscience, 2, 635-642.

O'Brien, E. J., \& Albrecht, J. E. (1992). Comprehension strategies in the development of a mental model. Journal of Experimental Psychology: Learning, Memory, \& Cognition, 18, 777-784. doi:10.1037/0278 $-7393.18 .4 .777$

Rajaram, S., \& Roediger, H. L., III (1993). Direct comparison of four implicit memory tests. Journal of Experimental Psychology: Learning, Memory, \& Cognition, 19, 765-776. doi:10.1037/0278 $-7393.19 .4 .765$

RANEY, G. E., \& RAYNER, K. (1995). Word frequency effects and eye movements during two readings of a text. Canadian Journal of Experimental Psychology, 49, 151-172. doi:10.1037/1196-1961.49.2.151

SCHACTER, D. L. (1994). Priming and multiple memory systems: Perceptual mechanisms of implicit memory. In D. L. Schacter \& E. Tulving (Eds.), Memory systems 1994 (pp. 233-268). Cambridge, MA: MIT Press.

TukeY, J. W. (1977). Exploratory data analysis. Reading, MA: AddisonWesley.

Westerman, D. L., Lloyd, M. E., \& Miller, J. K. (2002). The attribution of perceptual fluency in recognition memory: The role of expectation. Journal of Memory \& Language, 47, 607-617. doi:10.1016/ S0749-596X(02)00022-0

Wheeler, M. E., Petersen, S. E., \& Buckner, R. L. (2000). Memory's echo: Vivid remembering reactivates sensory-specific cortex. Proceedings of the National Academy of Sciences, 97, 11125-11129.

\section{APPENDIX \\ Experiment 1: Spoken/Spoken}

Story A
Introduction
Alex wanted to make some money to buy a new CD player. So she agreed to babysit her neighbor Kurt's dog
for the long weekend. After Kurt went over the instructions with Alex he said, "Damian is a wonderful dog. He'll
be great company for you."

\section{Critical Information}

Repeated Condition (Repetition Line):

Alex exhausted herself all weekend cleaning up after Damian. She tried to play with him, but he growled at her whenever she got too close. Alex had an early-morning class on Monday. Knowing that she would be gone when Kurt returned home, she left a voicemail on his phone. She mentioned that Damian was difficult to get along with and added: "Damian sure is great company. Nothing like being growled at for 48 hours."

\section{Not Repeated Condition:}

Alex enjoyed spending time with Damian over the weekend. They went for several peaceful walks in the park up the street. Alex had an early-morning class on Monday. Knowing that she would be gone when Kurt returned home, she left a voicemail on his phone. She mentioned that Damian is easy to get along with and added: "I really enjoyed babysitting Damian. I especially enjoyed taking him to the park." 


\section{APPENDIX (Continued)}

\section{Conclusion}

Then Alex headed off to her class. On the way there she stopped at the mini mart to get some coffee. She hoped today's class would be interesting.

\section{Story B}

\section{Introduction}

Justin had just moved to Baltimore to attend school at Johns Hopkins University. He hadn't been able to afford living alone, so he'd found a roommate. Today Justin spent most of the morning getting settled into his apartment, and then he went to buy the books he needed for his classes. Later that evening, Justin called his girlfriend, Kate, to fill her in on his day. He also told her all about his new roommate:

\section{Repetition Line}

"Wonderful guy, and he sure is great company."

\section{Disambiguation Line}

Justin found his roommate very annoying.

\section{Conclusion}

He knew that it could be very difficult to live with someone else, but his new roommate was the absolute worst.

(Manuscript received June 12, 2009;

revision accepted for publication October 28, 2009.) 